\title{
Oak-Hornbeam Forest Database of the Transylvanian Basin
}

\section{Anna Szabó}

Abstract: The report presents the structure and contents of a vegetation database of oak-hornbeam forests from the North-Western part of Romania (Transylvanian Basin). The Oak-Hornbeam Forest Database of the Transylvanian Basin (GIVD ID EU-RO-003) was developed to assemble published and unpublished relevés from the class Querco-Fagetea, mainly the Carpinion group from the study area. Currently it contains 524 relevés.

Keywords: phytosociology; relevé.

\section{GIVD Database ID: EU-RO-003}

\section{Oak-Hornbeam Forest Database of the Transylvanian Basin}

Scope: The database aims to compile phytosociological relevés of forests in North-Western part of Romania, Querco-Fagetea class, Carpinion group.

Status: completed and continuing

Period: 1941-2005

Database manager(s): Anna Szabó (annuc19@gmail.com)

Owner: (private)

Web address: [NA]

Availability: according to a specific agreement

Database format(s): TURBOVEG

Online upload: no

Publication: [NA]

Plot type(s): normal plots

Export format(s): TURBOVEG

Non-overlapping plots: 524

Plot-size range: $10-400 \mathrm{~m}^{2}$

Total plot observations: 524

Estimate of existing plots: [NA]

Number of sources: 16

Completeness: [NA]

Countries: RO: $100.0 \%$

Forest: [NA] — Non-forest: [NA]

Guilds: all vascular plants: $100 \%$

Environmental data: altitude: $66 \%$; slope aspect: $95 \%$; slope inclination: $86 \%$

Performance measure(s): cover: $100 \%$

Geographic localisation: small grid (not coarser than $10 \mathrm{~km}$ ): $100 \%$

Sampling periods: 1940-1949: 36.0\%; 1950-1959: 3.0\%; 1960-1969: 12.0\%; 1970-1979: 20.0\%; 1980-1989: 8.0\%; 1990-1999: 4.0\%; 2000-2009: $17.0 \%$

\section{Information as of 2012-07-12; further details and future updates available from http://www.givd.info/ID/EU-RO-003}

Anna Szabó (annuc19@gmail.com)

Transylvanian Carpathian Society Satu-Mare, Stefan cel Mare nr 6, 440114 Satu-Mare, ROMANIA 